\title{
Paul Valéry, penseur de l'attention et de la surprise
}

Julien Farges

\section{CpenEdition}

\section{Journals}

Édition électronique

URL : http://journals.openedition.org/alter/431

DOI : $10.4000 /$ alter.431

ISSN : 2558-7927

Éditeur :

Association ALTER, Archives Husserl (CNRS-UMR 8547)

\section{Édition imprimée}

Date de publication : 1 décembre 2016

Pagination : 169-194

ISBN : 978-2-9550449-2-6

ISSN : 1249-8947

\section{Référence électronique}

Julien Farges, " Paul Valéry, penseur de l'attention et de la surprise », Alter [En ligne], 24 | 2016, mis en ligne le 01 décembre 2017, consulté le 06 mai 2019. URL : http://journals.openedition.org/alter/431 ; DOI : 10.4000/alter.431 


\title{
PAUL VALÉRY, PENSEUR DE L'ATTENTION ET DE LA SURPRISE ${ }^{1}$
}

\author{
Julien Farges
}

S'il faut bien reconnaître que la présence de Paul Valéry est loin d'être envahissante dans le champ contemporain de la recherche philosophique, il est toutefois des thèmes et des problèmes relativement auxquels la pertinence de la référence à son œuvre ne saurait faire le moindre doute, malgré le caractère assurément et volontairement marginal de cette œuvre par rapport à l'histoire et à la pratique «officielle» de la philosophie. Or c'est un fait que la surprise fait partie de ces thèmes dans la mesure où elle représente une des expériences où s'atteste le plus clairement le dynamisme propre de la vie psychique. Car - faut-il le rappeler ? - à côté du poète, du théoricien de la création poétique et du brillant prosateur de circonstance, Valéry fut aussi et surtout l'homme d'une réflexion continue et secrète sur la structure et le fonctionnement de l'esprit, réflexion déposée dans les quelques 30000 pages de ses Cahiers, cet authentique «monde de pensées » que nous connaissons de moins en moins mal et que Valéry tenait pour son œuvre véritable, résultat et témoignage d'un effort intellectuel mené quotidiennement pendant plus de cinquante ans (entre 1894 et 1945$)^{2}$. Ce rappel suffit à faire com-

\footnotetext{
${ }^{1}$ Cet article est la version remaniée d'une communication faite le 25 mai 2015 dans le cadre du séminaire "Émotions et volitions » organisé par Natalie Depraz et Maria Gyemant aux Archives Husserl de Paris (UMR 8547, CNRS/ENS). Nous les remercions d'avoir fait une place à Valéry dans ce séminaire ainsi que pour leurs remarques sur ce travail.

${ }^{2}$ Les Cahiers de Valéry ont connu trois éditions correspondant à des principes distincts, auxquelles nous renverront selon les conventions suivantes : Cahiers, Paris, édition du CNRS en facsimile, 1957-1961, 29 vol. (désormais: C, suivi de la tomaison et de la pagination); Cahiers, anthologie thématique en deux volumes établie par J. Robinson-Valéry, Paris, Gallimard, « La Pléiade », 1973-1974 (désormais : Cahiers, suivi de la tomaison et de la pagination) ; Cahiers 1894-
} 
prendre que si la surprise fut un thème d'analyses et de descriptions pour Valéry, ce ne fut pas de façon pour ainsi dire autonome et directe mais seulement à titre de moment au sein d'une entreprise théorique plus globale qui concerne la vie de l'esprit dans toutes ses dimensions. Par conséquent, restituer la conception valéryenne de la surprise suppose de reconstruire au préalable les principes de la démarche théorique d'ensemble qui est celle de Valéry dans ses Cahiers afin de montrer à quelles conditions la surprise a pu devenir à ses yeux un thème de recherches à part entière. Ainsi pourra-ton rendre compte de l'originalité de la façon dont Valéry tente de $\mathrm{s}^{\prime}$ acquitter pour sa part et pour lui-même d'une tâche qui, pour le coup, n'a rien d'original, celle d'une connaissance scientifique du fonctionnement de l'esprit. Comment, en somme, faut-il concevoir l'esprit et son dynamisme propre pour que la surprise en devienne un révélateur privilégié ?

Pour répondre à cette question et disposer d'un fil conducteur dans cette reconstruction, il suffit de partir de ce que la surprise vient surprendre - à savoir une attente. Mais on voit rapidement que la relation conditionnelle qui s'esquisse ici possède une certaine complexité car la surprise ne se contente pas de supposer l'attente; elle prend en défaut cette attente qu'elle suppose, laquelle n'aura donc pas été assez attentive. Ainsi le couple attente/surprise semble-t-il impliquer une conception qui reconnaît dans le dynamisme attentionnel un caractère essentiel de la vie psychique. Or c'est bien ainsi qu'il en va chez Valéry, que l'on peut assurément inscrire dans la lignée des penseurs qui, dans le sillage de saint Augustin, voient en l'attention l'acte propre de l'esprit. Comme nous allons le montrer dans ce qui suit, c'est en effet en reconstruisant la conception valéryenne de l'attention qu'on peut se donner les moyens d'entrer dans les nombreuses analyses qu'il consacre au thème de la surprise, lequel se précise en retour comme une «rubrique » essentielle au sein de la théorie de l'attention - cette rubrique où le concept seulement fonctionnel de l'attention gagne chez Valéry une épaisseur quasiphénoménologique ${ }^{3}$.

1914, édition intégrale, Paris, Gallimard, 1987-2016, 13 vol. (désormais : CI, suivi de la tomaison et de la pagination).

${ }^{3}$ Cette thèse selon laquelle la surprise renvoie à une attention qui en conditionne non seulement la possibilité mais aussi la structure est aujourd'hui retrouvée et approfondie par Natalie Depraz dans la perspective d'une phénoménologie expérientielle à la croisée de la phénoménologie des émotions, des sciences de l'esprit et de la psychiatrie phénoménologique. Elle lui sert de fil conducteur aussi bien pour construire une phénoménologie expériencielle de la nouveauté («An experiential Phenomenogy of Novelty: The Dynamic Antinomy af Attention and Surprise", in Constructivist Foundation, vol. 8, n⿳3, 2013, p. 280-287), pour proposer les 
Ce faisant, cette étude espère également contribuer à montrer la fécondité de ce «travail sans nom $»^{4}$ que Valéry a inlassablement mené dans ses Cahiers et à le prémunir ainsi du double écueil qui nous semble menacer sa réception par les philosophes: le premier consiste à invoquer son œuvre littéraire et publique pour ne voir en Valéry qu'un poète s'étant risqué de façon plus ou moins dilettante et prétentieuse sur le terrain de la conceptualisation philosophique, le second à valoriser principalement la critique qu'il développe de la philosophie traditionnelle sur la base d'une analyse du langage pour l'accueillir dans le sérail philosophique au seul titre d'un "antiphilosophe $\aleph^{5}$, wittgensteinien avant l'heure, en laissant de côté de façon commode bien d'autres aspects de sa réflexion que l'intérêt des Cahiers est précisément de développer conjointement à la critique des illusions langagières - en particulier l'analyse toujours reprise du "moi pur» ou encore la corrélation fondamentale du corps, de l'esprit et du monde. Quoi qu'il en soit du caractère philo- ou misosophique ${ }^{6}$ de la pensée valéryenne, cette étude commencera par montrer quelle conception de l'attention se construit patiemment dans les pages des Cahiers afin de progresser à partir des conditions effectives de sa mise en œuvre volontaire vers les phénomènes de l'attente et de la surprise, couple notionnel où se montre peut-être de la façon la plus claire la conception originale et forte que Valéry se fait de la vie psychique et où, comme nous le verrons en fin de parcours, la signification de l'entreprise même des Cahiers vient se réfléchir.

I. 1. Pour comprendre comment Valéry en vient à l'attention comme thème fondamental de réflexion, d'analyse et de descriptions, et pour saisir le rôle considérable qui revient à cette notion dans sa pensée, deux facteurs peuvent être mentionnés, le premier étant structurel et le second conjoncturel. Le facteur structurel renvoie au cadre dans lequel se déploie le travail analytique de Valéry, c'est-à-

éléments d'une " cardiophénoménologie » («Cardiophénoménologie », in Cahiers philosophiques de Strasbourg, $\mathrm{n}^{\circ} 38,2015 \mid 2$, p. 47-83), que pour renouveler l'intérêt de la " première philosophie » de Paul Ricœur (" Attention et surprise. Paul Ricœur en débat et au-delà », in Alter. Revue de phénoménologie, $\mathrm{n}^{\circ} 23$, Dijon, Alter, 2015, p. 257-273, en particulier p. 267 sq.). Ces travaux confirment en retour la clairvoyance de Valéry et la fécondité de ses efforts descriptifs qu'il ne destinait pourtant qu'à un usage personnel, voire privé.

${ }^{4}$ C, XIII, p. 107.

${ }^{5}$ Cf. par exemple J. Bouveresse, «La philosophie d'un anti-philosophe : Paul Valéry » [1993], repris in Essais IV. Pourquoi pas des philosophes?, Marseille, Agone, 2004, p. 243-278 ; " De la philosophie considérée comme un sport », in Littérature, $\mathrm{n}^{\circ} 172$, "Paul Valéry, en théorie », décembre 2013, p. 85-119 ; C. Chauviré, Ludwig Wittgenstein, Paris, Seuil, 1989, p. 189-203.

${ }^{6} \mathrm{C}, \mathrm{XV}, \mathrm{p} .72$. 
dire au projet même des Cahiers. Comme on le sait, ces derniers représentent dans leur ensemble une vaste entreprise défensive, au départ engagée contre l'affectivité érotique, mais qui s'élargit bientôt en une réaction générale et acharnée contre l'emprise et la puissance de tout ce qui obscurcit l'esprit, y compris au sein de la vie intellectuelle: pas seulement l'affectivité, donc, mais aussi le vague, l'approximation, le spontané, l'arbitraire, la croyance, les prestiges du langage. Par opposition à tout cela, les Cahiers représentent une unique quête de lucidité où l'évaluation systématique des diverses opérations et productions de l'esprit dans leurs multiples relations doit déboucher sur la production de définitions et de conceptions précises et fiables.

Ce rappel sommaire suffit, avant toute définition de ce que Valéry entend par " esprit », " conscience » ou " connaissance », à faire apercevoir que l'attention est consubstantielle à l'entreprise des Cahiers elle-même tout d'abord en tant qu'exigence qui la commande. Si, comme Valéry le note dès l'un de ses premiers Cahiers, il y a un lien entre "ce qu'on appelle vie» et une certaine "quantité d'inattention $\gg^{7}$, et si la décision dont l'écriture des Cahiers est issue est celle d'une reconstruction volontaire de soi qui ne laisse pas au seul vital le soin de faire de moi ce que je suis, c'est manifestement que ces Cahiers peuvent être considérés à la fois comme le résultat et comme le lieu $d^{\prime} u n$ exercice volontaire ou encore d'une discipline de l'attention dont la fin est proche de l'idéal stoïcien de la maîtrise de soi ${ }^{8}$. Mais si les Cahiers impliquent, dans leur définition même, la notion d'attention, ce n'est pas seulement au titre de l'exigence disciplinaire factuelle d'où ils procèdent; $c^{\prime}$ est également, et de façon beaucoup plus décisive, au titre d'une condition essentielle de possibilité. Car si l'attention est une exigence méthodologique, c'est précisément parce qu'elle est le mode sur lequel l'esprit peut se connaître lui-même, la disposition dans laquelle l'esprit se rend accessible à l'esprit. C'est ce qu'indique Valéry lorsqu'il note en 1904 : «J'ignore mon esprit hors de l'attention ${ }^{9}$. Mais cette formule ne doit pas être mal comprise : elle signifie en réalité que c'est l'attention qui manifeste le plus proprement ce qu'on nomme l'esprit, qu'en elle l'esprit se donne à voir tel quel, même si l'attention est loin d'être la seule de ses fonctions. Autrement dit, c'est à partir de l'attention qu'il faut comprendre et définir l'esprit de façon dynamique et opératoire plutôt

\footnotetext{
${ }^{7}$ CI, I, p. 158.

${ }^{8}$ Cf. ibid., p. 230.

${ }_{9}$ CI, VI, p. 146.
} 
que de postuler à l'inverse une entité substantielle «esprit» qui aurait la propriété plus ou moins miraculeuse d'être attentive à soi.

C'est un point sur lequel nous reviendrons par la suite, mais plusieurs conséquences importantes s'en dégagent d'ores et déjà. En premier lieu, si une psychologie authentiquement scientifique doit être possible, elle ne le sera qu'à condition que soit déployée une attention concomitante ou de second degré - une attention à l'attention, de sorte que la thématique de l'attention ne devra plus être considérée comme une thématique spéciale, mais plutôt comme celle où se joue la possibilité même d'une psychologie. D'où cette remarque en 1913 : «L'analyse de l'attention est toujours chose capitale car elle décide entièrement de la valeur qui sera accordée à toute recherche intérieure $»^{10}$. Les deux dernières conséquences concernent quant à elles les Cahiers valéryens eux-mêmes : elles consistent à montrer que l'attention en fournit la véritable clé de lecture. En effet - deuxième conséquence - si c'est à partir de l'attention que l'esprit se laisse appréhender, cela implique que la totalité des notations des Cahiers concernant l'esprit ou l'une de ses fonctions (et peut-être même les poèmes en prose qui $\mathrm{s}^{\prime} \mathrm{y}$ esquissent de plus en plus fréquemment à partir de 1910) sont en un sens des produits de l'attention. En outre dernière conséquence - cela implique que les notations qui portent expressément sur l'attention doivent être lues sinon comme autant de définitions, du moins comme autant de descriptions convergentes de l'esprit lui-même. D’où, justement, l'extrême rareté des définitions explicites de ce dernier chez Valéry.

Si l'on comprend à présent dans quelle mesure le projet valéryen qui gouverne l'écriture des Cahiers était en un sens voué à rencontrer le thème de l'attention, qui traverse de fait les cinquante années de notations, il est un facteur conjoncturel ${ }^{11}$ qui a conduit à donner à ce thème une intensité particulière dans le travail de Valéry à partir de l'année 1903. L'Académie des sciences morales et politiques ayant choisi dès novembre 1902 l'attention comme sujet de concours pour l'attribution du prix Saintour de 1905 (section Philosophie), Valéry saisit l'occasion et se lance dans la rédaction d'un mémoire. Mais ne parvenant pas à ordonner la masse de ses remarques et analyses en suivant une ligne conductrice claire, il n'enverra finalement qu'un travail inachevé comprenant une note préliminaire et un premier chapitre, essentiellement introductif. Ce travail ne sera pas noté, et

${ }^{10}$ CI, XII, p. 219.

${ }_{11}$ Pour des indications historiques précises sur cet épisode cf. M. Jarrety, Paul Valéry, Paris, Fayard, 2008, p. 298-303 et CI, VI, p. 274-275 (note 1 de la p. 225). 
l'on ignore s'il a seulement été lu par le jury. Il a été édité pour la première fois en annexe du tome VI de $l^{\prime}$ «édition intégrale» des Cahiers, en 1997. Malgré cet échec, cet épisode a eu pour effet de fournir à la réflexion de Valéry un objet fixe de réflexion et d'analyse, qui envahit temporairement ses notes entre 1903 et 1905, le forçant à formuler de façon plus précise que de coutume sa pensée et sa méthode, à se les réapproprier, et orientant de façon décisive l'écriture des Cahiers jusqu'à la Première Guerre mondiale. C'est en nous appuyant essentiellement sur ce dossier de textes (Mémoire et Cahiers afférents) que nous allons maintenant présenter les éléments principaux de sa conception de l'attention.

I. 2. Comme nous l'avons indiqué dès le départ, le propre de la démarche de Valéry, dans ces textes, est de considérer l'attention comme un cas particulier au sein d'une théorie beaucoup plus large portant sur le fonctionnement de l'esprit. Le terme de "fonctionnement » est d'ailleurs à prendre ici en son sens fort car le premier point à souligner est que l'attention est pour Valéry l'une des principales fonctions de l'esprit, et non un phénomène : «L'attention n'est pas un phénomène, écrit-il ainsi. On la rend telle »-sous-entendu : par une attention exercée à son propre endroit ${ }^{12}$. L'attention serait ainsi la fonction produisant les phénomènes, c'est-à-dire donnant quelque chose à décrire sous le titre « esprit». Il est frappant de voir Valéry retrouver ici sans le savoir la distinction entre fonction et phénomène telle qu'elle structure la pensée de Carl Stump ${ }^{13}$, dont on sait quel théoricien de l'attention il fut. Mais force est de convenir que la proximité littérale cache une grande distance d'intention théorique car c'est l'indépendance relativement à la variation de son objet qui fait la fonctionnalité de la fonction chez Valéry: "Quel que soit l'objet de l'attention, les formes physiques et psychiques demeurent à peu près les mêmes $[. .$.$] - Encore un invariant { }^{14}$. En continuité avec cette première approche de l'attention, l'esprit lui-même ne sera donc rien d'autre pour Valéry qu'un ensemble structuré de fonctions, de sorte que sa théorisation scientifique consistera à mettre au jour ces fonctions dans leurs relations fondamentales de dépendance ou d'indépendance.

\footnotetext{
${ }^{12}$ CI, VI, p. 127.

${ }^{13}$ Cf. C. Stumpf, «Phénomènes et fonctions psychiques » [1906], trad. D. Fisette in Renaissance de la philosophie. Quatre articles, Paris, Vrin, 2006, p. 133-167.

${ }_{14}$ CI, VI, p. 157. Cf. également p. 169 : «L'attention ne dépend pas de son objet » ; CI, II, p. 106.
} 
Conformément à cette perspective, et malgré son invariance relativement à ses objets, il est important de souligner avec les éditeurs $\mathrm{du}$ tome VI des Cahiers que l'attention comme fonction sera considérée par Valéry dans le cadre de la représentation des fonctions totales de l'esprit et comme l'une de ses variables essentielles, constitutives de son fonctionnement $\mathrm{d}^{\prime}$ ensemble ${ }^{15}$. C'est pourquoi il insiste sur le fait que l'attention suppose et implique à titre de condition de possibilité la variabilité du système mental, cette variabilité fondamentale de la vie psychique qu'il baptise «self-variance ». Et de fait, ce que Valéry nomme la " mise en attention ${ }^{16}$ correspond au simple pouvoir de «se tourner vers », indépendamment de l'objet; l'attention s'identifie donc à la "mobilité interne » ou encore au «summum $\mathrm{du}$ multivoque $»^{17}$. On serait tenté cette fois de rapprocher cette description de la conception intentionnaliste de la Zuwendung développée par Husserl au même moment ${ }^{18}$; mais là encore, la proximité n'est qu'apparente puisque la dépendance de l'attention relativement à la variabilité du système mental signifie surtout pour Valéry que ce dernier est susceptible d'être réorganisé par elle et les relations entre ses autres fonctions reconfigurées à partir d'elle. On pourrait dire ainsi de l'attention qu'elle est chez Valéry la réponse fonctionnelle de l'esprit à sa self-variance, et le point remarquable est que cette réponse, cette réorganisation, est essentiellement du type d'une fixation de ce qui est variable et mobile.

Ce terme mérite qu'on s'y arrête ; il n'est certes pas souvent utilisé dans les Cahiers ${ }^{19}$, mais c'est qu'il s'efface souvent derrière ses modalités, décrites par Valéry tantôt négativement, tantôt positivement. Négativement, la fixation opérée par l'attention est décrite comme une restriction, " restriction de notre variabilité totale $»^{20}$ qui n'annule donc pas la variation comme telle: "Fixer l'esprit sur un objet n'est pas immobiliser - C'est restreindre la variance - le champ de variation de l'esprit ${ }^{21}$. Il pourrait sembler que Valéry se contente ainsi d'exploiter l'opposition convenue entre attention et distraction, et il est vrai qu'il lui arrive de noter à propos de l'attention qu'elle est

\footnotetext{
${ }^{15}$ Cf. VI, p. 263 (note 1 de la p. 113).

${ }^{16}$ CI, IV, p. 166 ; cf. aussi CI, III, p. 540.

${ }^{17}$ CI, VI, p. 130.

${ }^{18}$ Cf. par ex. E. Husserl, Phénoménologie de l'attention, trad. N. Depraz, Paris, Vrin, 2009, «Directions de l'attention », p. 203 sq. (Hua XXXVIII, p. 371 sq.).

${ }^{19}$ Cf. par ex. CI, VI, p. 22, p. 123 ; CI, III, p. 87, p. 135.

${ }^{20}$ CI, VIII, p. $88 ; C I$, VII, p. 168.

${ }^{21}$ CI, VI, p. 123. Cf. également CI, IV, p. 106 : «l'attention, la résistance à l'inertie de la selfvariance ».
} 
«écartement de distractions $»^{22}$. Mais une formule remarquable permet d'aller plus loin dans l'analyse : en contradiction avec ce qui précède, il note dans le même cahier: "L'attention est une distraction $»^{23}$. En réalité, la contradiction n'est qu'apparente car ce que cette formule provocante cherche à souligner, c'est que congédier les distractions revient exactement à se distraire d'elles, à s'en détourner. C'est finalement la même ambiguïté étymologique que celle du divertissement chez Pascal, qui est bien une conduite défensive. Précisément, Valéry attribue explicitement une fonction défensive à l'attention, laquelle s'active essentiellement contre, au sens où son action est finalement toujours une réaction: "contre l'écoulement nécessaire des faits mentaux » elle retient, et «contre la diversité et $l^{\prime}$ instabilité $»^{24}$ elle stabilise. Comme on le voit aisément, c'est un point de vue énergétique, proche des conceptions positivistes de Mach (dont Valéry a probablement lu La connaissance et l'erreur, traduit en français en $1908^{25}$ ), qui domine ici la description : énergétiquement, l'attention vaut la distraction. Or l'intérêt de ce point de vue est aussi de valoriser le rôle paradoxal des obstacles dans la stimulation de l'attention $^{26}$, ce qui ouvre ainsi la description valéryenne aux conditions effectives et concrètes de sa mise en œuvre et conduit à la définition de l'esprit attentif comme «système gêné » ${ }^{27}$.

Mais cette puissance de fixation du variable qu'est l'attention fait également l'objet d'une caractérisation positive, qu'on peut approcher par le moyen de l'opposition entre l'ordre et le chaos: Valéry voit dans l'attention la capacité à «débrouiller le chaos » ${ }^{28}$ des faits de consciences et des états mentaux qui, tels qu'ils sont vécus, forment une incohérence qui échappe à la conscience elle-même. En ce sens, l'attention fonctionne comme révélateur pour l'esprit de sa propre inintelligibilité initiale. Mais soucieux de précision analytique dans ses descriptions, Valéry ne se contente pas de l'image d'un chaos à débrouiller ; au contraire, il ressaisit l'opération propre de l'attention à partir d'une distinction notionnelle qui lui est propre et qui traverse l'ensemble des Cahiers - la distinction entre le formel et le significatif. Sans cesse réexposée, de façon plus ou moins développée, plus ou

22 CI, VI, p. 192.

${ }^{23}$ Ibid., p. 122.

${ }^{24}$ Ibid., respectivement p. 126, p. 165. Cf. aussi p. 133 à propos de la stabilisation; p. 178 où il est question d'une «élimination » de l'inutile ; CI, IV, p. 195, d'une « annulation de certains changements »; p. 221 d'une "négligence de ce qui n'importe pas ».

${ }^{25}$ E. Mach, La connaissance et l'erreur, trad. M. Dufour, Paris, Flammarion, 1908.

${ }^{26}$ CI, VI, p. 163, p. 204.

27 Ibid., p. $146-147$.

${ }^{28}$ Ibid., p. 78 et p. 130. 
moins claire, cette distinction passe en gros entre l'esprit tel qu'il est donné et l'esprit tel qu'il est connu. Le point décisif est évidemment ici que l'esprit ne peut être connu tel qu'il est donné et que cette distinction fait donc apercevoir le caractère principiellement nonphénoménologique de la démarche valéryenne, mais plutôt son orientation résolument constructiviste.

Le formel renvoie à la vie mentale telle qu'elle est vécue ou telle qu'elle se donne à vivre, c'est-à-dire au fait en lui-même insignifiant et immédiat de la succession uniforme et indéfinie des états mentaux ${ }^{29}$. Succession et uniformité sont les deux caractères essentiels ici, de sorte que Valéry peut nommer « lois formelles [...] celles à l'égard desquelles les événements de la conscience peuvent être regardés comme équivalents, comme des unités qui se substituent $»^{30}$. Comme exemple d'une telle loi il donne la nécessité de l'accroissement incessant des états mentaux, ou encore la nécessité du changement dans la conscience. Ce sont des lois qui font donc abstraction de la particularité des vécus qui s'enchaînent dans la conscience, et a fortiori de ce qui s'y trouve vécu. Par opposition, les lois significatives sont celles «qui de nos éléments [...] forment des moyens, des intermédiaires, des groupes ${ }^{31}$. Or il faut souligner qu'aucune série d'états ou de vécus n'est significative par elle-même ${ }^{32}$ : le significatif ne peut qu'être produit par une intervention ou une opération sur le formel, si bien qu'il désigne comme tel le milieu et le résultat de ce qu'on nomme la connaissance ${ }^{33}$ et que les nécessités significatives sont proprement celles du connaître et non de l'être. Or la présence du concept de groupe dans la citation précédente, qui s'oppose à l'élémentarité formelle des états mentaux, permet de comprendre que les caractères du significatif seront pour Valéry opposés terme à terme à ceux du formel: le significatif sera établi par production de simultanéité au lieu de succession, du connexe au lieu du linéaire, du contrasté au lieu de l'uniforme, du fini au lieu de l'indéfini, de la répétition au lieu de l'instantanéité, du réversible au lieu de l'irréversible, etc. ${ }^{34}$ Dans ce contexte, c'est l'opération de substitution qui est l'agent de cette production: "le significatif, substituer dans quelque chose, la partie au tout, l'instantané à la durée, etc. » ${ }^{35}$ Une

\footnotetext{
${ }^{29}$ Cf. ibid., VI, p. 84 ; CI, XII, p. 309.

${ }^{30} \mathrm{CI}, \mathrm{VI}, \mathrm{p} .228$.

${ }^{31} \mathrm{Idem}$.

32 Ibid., p. 104.

${ }_{33}$ Cf. ibid., p. 38.

${ }^{34}$ Cf. ibid., p. 84 et XII, p. 219.

35 Ibid., p. 309.
} 
conclusion s'impose dès lors: produire du significatif, $c^{\prime}$ est très exactement modéliser, c'est-à-dire construire une représentation stable d'un système intrinsèquement variable, en lui-même insignifiant. L'essentiel du travail théorique auquel se livre Valéry dans les milliers de pages de ses Cahiers peut donc être décrit comme un travail de dégagement de ce qu'il y a de significatif dans la vie de l'esprit ainsi que des modes de sa production ${ }^{36}$.

Or tous les éléments précédents se nouent dans la mesure où la modalité positive selon laquelle Valéry ressaisit la puissance de fixation qu'est l'attention consiste précisément à voir en elle la production du significatif, autrement dit - et de nouveau de façon négative, par opposition au formel - la propriété fondamentale de «n'être pas régi par les événements purs ${ }^{37}$. Nous touchons là au cœur de la conception valéryenne de l'attention : l'attention comme création, formation ou production du significatif - cette formule est constamment répétée dans les Cahiers, parfois comme une sorte de point de repère auquel Valéry revient lorsqu'il lui arrive de se perdre dans des analyses partielles ou locales ${ }^{38}$. Mais il faut comprendre que sous cette forme, cette formule est encore incomplète, comme le montre une notation de 1899 dans laquelle, après avoir défini les propriétés significatives comme "propriétés de la relation hors de la succession ", Valéry passe aux propriétés formelles au premier rang desquelles il place l'attention ${ }^{39}$. Y a-t-il contradiction avec ce qui précède? Non. Il nous semble que c'est seulement le biais par lequel Valéry retrouve la distinction topique de la psychologie de l'époque entre attention spontanée et attention volontaire : l'attention est bien d'abord un état parmi d'autres au sein de la vie psychique, qui peut succéder à d'autres sans être porteur d'une volonté explicite de saisie mais simple-

\footnotetext{
${ }^{36}$ Il nous semble donc que les éditeurs du tome X de l'édition intégrale des Cahiers commettent un contresens regrettable à propos de la distinction valéryenne du formel et du significatif lorsqu'ils écrivent (CI, X, p. 467) : «ce qui intéresse l'auteur des Cahiers, c'est le fonctionnement de l'homme quelconque et non l'histoire d'un individu particulier, le formel et non le significatif. Il cherche des lois générales ». Assurément, Valéry est à la recherche des lois générales de fonctionnement de l'esprit, ce qui le pousse à penser « en quelconque ", selon l'une de ses formules ; mais le significatif et le formel désignent l'un et l'autre des régimes parfaitement généraux de fonctionnement de l'esprit, correspondant à deux régimes distincts de variation : la pure et simple selfvariance dans le premier cas, insignifiante comme telle, et la production de variations et d'invariants signifiants par transformation et substitution dans l'autre cas. La superposition de la distinction général/particulier à la distinction formel/significatif confond en réalité le significatif avec ce que Valéry appelle l'accidentel (c'est-à-dire précisément le particulier dans sa contingence : cf. CI, XII, p. 309-310) et cette méconnaissance du significatif conduit inévitablement à plaquer sur la recherche de Valéry ce formalisme dont il a toujours cherché à se garder.

${ }^{37}$ CI, VI, p. 114.

${ }^{38}$ Cf. par exemple CI, III, p. 239 ; IV, p. 216 ; VI, p. 92, p. 160, p. 182 ; VII, p. 329 ; XI, p. 284.

${ }^{39}$ CI, III, p. 239.
} 
ment comme un réflexe en réponse à des stimulations sensibles de notre environnement; mais, dirigée par la volonté, elle devient l'opérateur qui s'abstrait du formel pour produire le significatif. La formule la plus précise pour définir l'attention serait donc la suivante, de 1904 : «état formel pour faire du significatif $»^{40}$.

Si l'on rattache ce résultat à l'idée, introduite plus haut, selon laquelle c'est à partir de l'attention qu'il faut définir l'esprit, on parvient à la thèse selon laquelle, loin de tout spiritualisme, ce dernier n'est autre que l'instance de la production attentive du significatif, c'est-à-dire puissance de transformation attentive d'un donné initial formel et insignifiant. C'est ce que confirme le texte suivant, où Valéry livre l'une des rares définitions de ce qu'il entend par « esprit »:

Par ce nom d'esprit, je n'entends pas du tout une entité métaphysique; j'entends ici, très simplement, une puissance de transformation que nous pouvons isoler, distinguer de toutes les autres, en considérant simplement certains effets autour de nous, certaines modification du milieu qui nous entoure et que nous ne pouvons attribuer qu'à une action très différente de celle des énergies connues de la nature; car elle consiste au contraire à opposer les unes aux autres ces énergies qui nous sont données ou bien à les conjuguer ${ }^{41}$.

Et dans la mesure où l'attention est l'acte par lequel l'esprit devient visible pour lui-même comme une telle puissance, on peut dire de lui qu'il est à la fois le sujet et l'objet de l'attention et déclarer de cette dernière qu'elle est à tous égards pour Valéry le lieu de l'esprit $^{42}$.

I. 3. Mais un dernier aspect de la réflexion valéryenne sur l'attention reste à mentionner. En effet, quand bien même l'attention est la fonction par laquelle l'esprit rend significatif y compris ce tout variable qu'il est lui-même, il est possible d'en affiner la compréhension en la soumettant elle-même à des modèles qui permettent d'en décrire,

\footnotetext{
${ }^{40}$ CI, VII, p. 96.

${ }^{41} \mathrm{P}$. Valéry, «La politique de l'esprit. Notre souverain bien» [1932], repris dans Variété in CEuvres, Paris, Gallimard, « La Pléiade », 1957, tome I, p. 1014-1040, ici p. 1022.

${ }^{42}$ Cette lecture serait confirmée par le fait qu'il arrive à Valéry de donner à l'attention et à l'esprit lui-même les mêmes caractéristiques fonctionnelles ou opératoires. C'est d'abord le cas avec la transformation elle-même (CI, VI, p. 121), mais c'est aussi le cas avec la combinaison (qui est à vrai dire un cas de transformation) dans une série de notations datant de $1901: l^{\prime}$ esprit s'y trouve d'abord déterminé comme puissance insatiable de combinaison ( $C I, I V$, p. 172), puis, quelques pages plus loin: "l'attention est la combinaison» (p. 177). Une même conclusion s'impose : l'attention est l'acte le plus authentique de l'esprit ou l'esprit en acte lui-même.
} 
certes indirectement mais de façon plus précise, le fonctionnement. Trois modèles principaux sont à l'œuvre dans les Cahiers de Valéry à l'époque du Mémoire inachevé, que nous allons présenter rapidement en montrant que leur grand intérêt est de mettre progressivement la problématique de l'attention en relation avec la temporalité et, par là, avec la thématique de la surprise.

Le premier modèle est celui de l'accommodation visuelle, parfaitement exprimé par Valéry dans l'analogie suivante: "L'attention est à la perception générale ce que l'accommodation est à la rétine "- que cette dernière soit consciente ou non ${ }^{43}$. L'analogie suggère tout d'abord qu'on peut se représenter l'attention comme un regard particulier opposé au simple voir inattentif de la conscience immédiate. Mais elle suggère aussi que la "mise en attention » de l'objet correspond à une coordination dynamique de plusieurs opérations dont le résultat est une vision nette susceptible de se prolonger malgré la poursuite de variations concomitantes ${ }^{44}$. Le point intéressant est ici que l'attention est stérile si elle demeure ponctuelle ou aussi instantanée qu'un état formel; c'est au contraire sa capacité à durer dans le temps qui la rend capable de produire le significatif par substitution et transformation. Cette conception analogique de $l^{\prime}$ attention comme "accommodation mentale $»^{45}$ appelle dès lors son prolongement à travers deux autres types de descriptions, qui correspondent à deux autres types de modélisation : soit des descriptions énergétistes ou économiques, propres à faire droit à l'effort que représente l'attention et qu'il faut soutenir ${ }^{46}$ dans le temps; soit des descriptions de l'esprit comme système temporairement attentif tendant à se conserver dans cet état.

Les descriptions qui font de l'attention « une forme d'énergie ${ }^{47}$ renvoient quant à elles au modèle de la tension musculaire, ce qui conduit Valéry à parler de "tension mentale », rapprochée par analogie de la «contraction musculaire», jusqu'à oser le raccourci suivant : «l'attention - musculature de l'esprit ${ }^{48}$. Ce modèle pousse dès lors à chercher un analogon de la contraction physique que Valéry semble trouver dans la contention de l'esprit ${ }^{49}$, qui lui permet de

\footnotetext{
${ }^{43}$ CI, VII, p. 169.

${ }^{44}$ Cf. CI, VI, p. 117 ; C, XXVII, p. 740, où Valéry parle d'un « système de coordination tend[ant] à conserver la netteté ».

45 CI, VI, p. 87.

${ }^{46}$ Cf. ibid., p. 140 : «Soutenir l'attention! ».

${ }^{47}$ CI, IV, p. 294. Cf. aussi VI, p. 49.

${ }^{48}$ Ibid., respectivement p. 118, p 135, p 180.

49 Ibid., p. 121.
} 
retrouver certains aspects des philosophies de l'effort intellectuel, telles qu'elles font droit aux phénomènes de résistance. Mais surtout, il permet de situer l'attention au point d'articulation du physique et du psychique, de regarder les efforts de l'attention comme étant en eux-mêmes les préparations ou les amorces d'actions corporelles, jusqu'à affirmer, dans une étonnante proximité avec la pensée de Mach, que l'attention est le point depuis lequel la distinction entre le physique et le psychique devient relative ${ }^{50}$. Enfin, dans la mesure où il permet de rendre compte de la possibilité d'une acquisition progressive de l'attention, de son développement par une discipline et des exercices qui seraient les analogues d'une gymnastique ou d'un entraînement ${ }^{51}$, ce modèle l'inscrit dans une durée plus large que celle de sa mise en œuvre effective: il l'inscrit dans ce qu'on pourrait appeler une «histoire de l'esprit » dont le terme serait la maîtrise de soi. À cet égard, il ne fait guère de doute que les Cahiers, dans leur ensemble, formaient pour Valéry lui-même le terrain d'exercice dans les deux sens du terme - de son attention.

Enfin, les descriptions de l'attention comme état dans lequel l'esprit se trouve et cherche à se maintenir renvoient à une troisième modélisation, d'origine thermodynamique, mobilisant le concept de " phase ». Dans cette perspective le dynamisme de l'esprit appelle l'idée d'une théorie des phases, c'est-à-dire, dans les termes de Valéry, "des transformations sensitivo-mentales dans lesquelles le système vivant-sentant-pensant peut se regarder comme un système isolable ${ }^{52}$. L'attention est l'une de ces phases, plus précisément elle est celle « où l'ordre des représentations dépend de leur nature »53, ce qui n'est bien sûr jamais le cas dans la succession formelle immédiate des états mentaux. Comme on le voit, le grand intérêt de cette modélisation de l'attention comme phase est de mettre au centre son caractère intrinsèquement instable $d^{\prime}$ ' état transitoire $d^{\prime}$ un système ${ }^{54}$ et, comme tel, temporaire. Ce point est fondamental en effet car il nous semble que c'est lui qui ouvre les Cahiers valéryens à une prise en considération pleine et entière de la temporalité concrète et incarnée de l'attention - à partir de laquelle elle s'articule au couple attente/surprise.

\footnotetext{
${ }^{50}$ Ibid., p. 29. Cf. E. Mach, L'analyse des sensations. Le rapport du physique au psychique, trad. F. Eggers et J.-M. Monnoyer, Nîmes, Jacqueline Chambon, 1996, p. 20.

${ }^{51}$ Cf. CI, VI, p. 178.

${ }^{52}$ C, XXVII, p. 739-740.

${ }_{53}$ CI, VI, p. 208. Cf. aussi p. 174.

54 Ibid., p. 113.
} 
II. 1. Pour le montrer, arrêtons-nous tout d'abord sur la relation de l'attention au temps, relation complexe sont Valéry montre qu'elle suppose que plusieurs niveaux de temporalité soient distingués. Partons du résultat de l'attention, qui est de «rendre présent »; il nous semble que cette expression doit être prise au pied de la lettre, au sens où l'attention produit le présent. De même façon, quand Valéry écrit «le présent est ce à quoi revient toujours l'attention »55, il ne veut pas dire qu'elle serait liée à cette dimension du temps en particulier, qui lui préexisterait et au sein de laquelle elle opérerait, mais bien que l'attention institue au contraire cette dimension. Pour comprendre ce qui est en jeu ici, il faut revenir à l'opposition du formel et du significatif : en tant que formelle, la succession immédiate des états psychiques est une temporalité insignifiante, écoulement plutôt que passage. Le passage significatif du temps suppose qu'un élément du système soit fixé et que le reste s'ordonne par rapport à lui dans des relations qui sont dès lors d'antériorité, de postériorité ou de simultanéité. C'est du moins ce qu'il semble que Valéry veuille dire lorsqu'il déclare qu' « on ne perçoit le temps que par l'attention ", thèse qu'il formule parfois de façon plus condensée en disant que «le temps n'est au fond que de l'attention $»^{56}$. Si le temps est ainsi l'un de ces produits significatifs de l'attention, il est impossible de dire de l'attention qu'elle prend place dans le temps ; mais puisque l'attention est en même temps une phase, un état transitoire de l'esprit, elle a néanmoins une durée qui lui est propre, délimitée par un commencement et par une fin et passant elle-même par des phases ${ }^{57}$.

Or si nous avons soutenu plus haut que la démarche de Valéry était, dans ses principes, non phénoménologique, il semble qu'une dimension phénoménologique se fait tout de même jour dans les notations relatives à l'expérience de l'attention, en particulier à partir de sa fin, de son interruption, dimension qui entre en tension avec l'orientation constructiviste : car si, d'un côté, seule une attention au second degré, concomitante et comme parallèle à une attention de

\footnotetext{
${ }^{55}$ Respectivement ibid., p. 129 et VIII, p 205.

${ }_{56} \mathrm{CI}$, VI, respectivement p 186 et p 140. Cf. aussi p. 162 ; XI, p 346 : «ce présent, l'accommodation actuelle ». Ces notations font évidemment entrevoir l'immense intérêt d'une confrontation systématique entre cette conception et celle que développe saint Augustin notamment dans le livre XI des Confessions où, comme on le sait, le lien entre attention et mémoire du présent s'approfondit en une description de la conscience du temps selon ses trois dimensions. Ainsi se trouverait documenté le sentiment de proximité que reconnut Valéry avec les réflexions de l'évêque d'Hippone lorsqu'il en lut les Confessions, vraisemblablement en 1901 (cf. M. Jarrety, Paul Valéry, op. cit., p. 309).

57 CI, VI, p 121, p 178.
} 
premier degré permet de la modéliser et rend au fond possible, comme on l'a vu, la psychologie comme science, il reste d'un autre côté, que nous ne pouvons rendre compte de notre expérience effective de l'attention que de l'extérieur et de façon différentielle car elle est "un état dont ne s'aperçoit que lorsqu'on en sort ou qu'il faiblit $»^{58}$. S'il y a donc une phénoménologie valéryenne de l'attention, c'est une phénoménologie des limites de l'attention, à l'occasion de laquelle ce n'est plus tant d'un esprit insulaire qu'il est question mais de ce que Valéry appellera bientôt le « système C-E-M » (corpsesprit-monde) ${ }^{59}$. En effet, les limites de l'attention sont évidemment reconductibles aux restrictions qui nous sont imposées par notre condition de vivants incarnés et inscrits comme tels dans une dépendance multiforme relativement au monde. Ces restrictions sont principalement de deux sortes pour Valéry et sont exprimées par lui dans la formule synthétique «ni longtemps, ni à la fois ${ }^{60}$. Cette dernière restriction concerne bien entendu le fait que notre attention ne peut pas se porter actuellement sur toute chose même si elle s'étend potentiellement à toute chose ${ }^{61}$. Quant à la première restriction, concernant la durée de l'attention, elle débouche à son tour sur deux types de limites: notre attention ne dure "pas toujours - ni continûment ${ }^{62}$. La continuité est compromise par les multiples sollicitations et perturbations issues du monde et relayées par notre sensibilité ; et la continuation indéfinie est rendue impossible par le fait que l'attention est un effort, c'est-à-dire par le phénomène de la fatigue. Ce n'est pas le moindre des intérêts des travaux valéryens sur l'attention, en effet, que de ménager une place au rôle que joue la fatigue relativement à elle, rôle double à vrai dire, de part et d'autre de l'attention, et que nous ne pouvons que mentionner ici : la fatigue est tout d'abord consécutive à l'attention dont elle vient amoindrir l'efficacité au cours de sa mise en œuvre et finalement interrompre temporairement l'activité ; mais à l'inverse l'attention est en un sens consécutive à la fatigue dans la mesure où c'est cette dernière qui

\footnotetext{
58 Ibid., p. 141.

${ }^{59}$ Sa première occurrence date de 1921 et se trouve en C, VIII, p 203 (repris in Cahiers, I, p. 1126). Pour une généalogie de cette triade, cf. J. Schmidt-Radefelt, «Valéry face aux présences de Corps/ Esprit/Monde (CEM) », in Paul Valéry contemporain, Saint-Clément-de-Rivière, Fata Morgana, 2013, p. 105-118.

${ }^{60}$ Cf., entre autres formulations, CI, VII, p 99 ( = C, III, p 288 ; repris in Cahiers, I, p. 897) ; C, XVI, p 266 (repris in Cahiers, I, p. 1035) ; C, XXVI, p 719 (repris in Cahiers, I, p. 856)

${ }^{61}$ Cf. CI, VI, respectivement, p. 113 et II, p. 106.

${ }^{62}$ CI, VI, p. 194.
} 
nous fait connaître en premier lieu et après coup l'attention comme un effort ${ }^{63}$.

Le résultat de ces analyses de l'expérience incarnée de l'attention tient dans la définition paradoxale de sa durée propre comme « continuité intermittente $»^{64},-$ expression aporétique qui cherche à nommer à la fois l'attention comme trame de fond de notre vie et les perturbations qui la travaillent de l'intérieur sans introduire en elle de réelle discontinuité. Or c'est justement à partir de cette définition paradoxale qu'on peut voir comment la double thématique de l'attente et de la surprise vient s'agréger chez Valéry à la thématique de l'attention et l'enrichir de nouvelles dimensions, que nous allons reconstruire à présent.

II. 2. L'intense travail d'analyse et de réflexion sur l'attente et la surprise auquel se livre Valéry entre 1912 et 1915 sur la base de notations antérieures ${ }^{65}$ nous paraît en effet le prolongement naturel de son travail «quasi-phénoménologique »sur l'attention envisagée dans sa durée spécifique dans la mesure où ce couple notionnel n'est finalement que le moyen de déployer en des analyses précises l'idée d'une "continuité intermittente ». L'attention vécue se spécifie en quelque sorte à travers le jeu de l'attente et de la surprise, l'attente prenant en charge l'élément de continuité n'excluant pas des interruptions et la surprise nommant ces interruptions qui procèdent de la continuité elle-même. Certes, les analyses valéryennes de l'attention en 1903-1904 laissent souvent présager ce lien avec la thématique attente/surprise, comme lorsque l'attention est mise en équivalence avec une anticipation, une protention, ou bien quand Valéry déplie le concept d'attention en une " attente tendue ${ }^{66}$. Mais rien ne vaut en richesse de perspectives le dossier de textes de 1912-1915, où l'étude $\mathrm{du}$ couple attente/surprise le conduit à une recherche foisonnante concernant la continuité et la discontinuité dans la vie mentale : dans ces pages, Valéry tente de cerner ce qu'il nomme le « rythme » propre de cette vie, sa scansion intime, c'est-à-dire la façon dont sa continuité exclut toute linéarité mais procède de et se donne à travers des « crises", des «événements» ou d'irruptions de «nouveauté » ${ }^{67}$. Pour exposer les principaux résultats qui se dégagent de cette masse de notations disparates, on peut prendre pour fil conducteur la

\footnotetext{
63 Pour ces deux aspects, cf. respectivement ibid., p. 119, p. 120, p. 130, p. 146 ; IV, p. 294.

${ }^{64} \mathrm{CI}, \mathrm{VI}, \mathrm{p} .164$.

${ }^{65} \mathrm{Ce}$ « dossier » a été récemment (2009) reconstitué et édité in CI, XI, p. 255-364.

${ }_{66} \mathrm{Cf}$. CI, VI, respectivement p. 69, p. 205 et p. 113.

${ }^{67}$ Cf. CI, XI, p. 268 (crise), p. 277, p. 322 (événement), p. 266, p. 285, p. 289 (nouveauté).
} 
tension circulaire qui s'installe entre l'attente et la surprise, rendue manifeste par deux formules importantes, relevées à fort juste titre par les éditeurs du tome XI des Cahiers ${ }^{68}$. Voici la première de ces formules : "Jamais mon attente n'est assez complète, mes conditions connues assez nombreuses pour que je défie toute Surprise»; en d'autres termes: l'attente a beau être un phénomène structurant ma vie, elle ne peut exclure totalement la surprise puisqu'au contraire elle la rend possible, de sorte que la surprise est en réalité à la mesure de mon attente et que "la surprise est toujours possible ${ }^{69}$. La deuxième formule, qui entre en tension avec la précédente, déclare quant à elle: "Précieuse est la surprise qui fait découvrir l'attente générale $»^{70}$; en $\mathrm{d}^{\prime}$ autres termes, la surprise remplit ici une fonction révélatrice de ce qui la rend possible, de façon analogue au rôle que remplit la fatigue pour l'attention. Chacune des deux notions rend donc l'autre possible, de sorte qu'on peut traiter de l'une indépendamment de l'autre ${ }^{71}$; c'est ce cercle que nous allons parcourir dans ce qui suit, en insistant sur le fait qu'il ne permet pas seulement de rendre compte de la continuité paradoxale de notre vie mentale mais aussi de sa fécondité propre, de sa capacité à produire quelque chose comme une connaissance, comme le montre cette notation qu'on dirait échappée aux Pensées de Pascal et où le couple attente/surprise se superpose de façon éloquente au couple attention/distraction :

Sans l'obligation de nous tourner vers l'événement inattendu ou l'idée adventice, nous serions à la merci de notre pensée commencée. La menace la plus grave nous y laisserait.

Et sans la force de maintenir cette pensée pour un temps, nous serions à la merci du moindre bruit, du moindre passé, du hasard continuel.

DONC, sans distraction, danger. Sans attention, impuissance. Aveugle ou paralytique ${ }^{72}$.

\footnotetext{
${ }^{68}$ Cf. ibid., p. 414-415 (note 1 de la p. 257).

${ }^{69} \mathrm{Ibid}$., respectivement p. 260 et p. 285 (cf. aussi p. 303).

${ }^{70} \mathrm{Ibid} ., \mathrm{p} .341$.

${ }^{71} C^{\prime}$ est cette circularité dont Natalie Depraz cherche à approfondir la sens et la structure dans ses travaux récents sous le titre de «l'antinomie dynamique de l'attention et de la surprise » («An experiential Phenomenogy of Novelty: The Dynamic Antinomy af Attention and Surprise», art. cit., p. 284). Une fois précisées les modalités selon lesquelles l'attention se spécifie en «vigilance ", une double relation de conditionnement est mise en évidence : "pas de surprise sans attention-vigilance » (idem.), " pas d'attention-vigilance sans la réalité de la surprise » (ibid., p. 285). ${ }^{72}$ CI, XI, p. 357.
} 
Commençons donc par ce qui nous fait échapper à la paralysie, cette attention conçue comme «attente tendue». Il ne s'agit pas, tout d'abord, de l'attention volontaire mais d'un phénomène pour ainsi dire spontané, propre à la vie psychique comme telle et formant pour ainsi dire sa trame de fond, une sorte $d^{\prime}$ " attention continue » ou $\mathrm{d}^{\prime}$ « accommodation permanente moyenne ${ }^{73}$ que Valéry nomme " attente générale»: " "Ce que je suis" est une attente permanente, générale [...]. Nous vivons dans une préparation ou une disposition perpétuelle $»^{74}$. C'est cette attente qui fait ce que Valéry nomme «ma continuité ${ }^{75}$, qui est tout autant continuité de l'être que du connaître et à propos de laquelle on peut souligner trois points. D'une part le fait que, fidèle à sa méthode de modélisation analogique, il arrive à Valéry de recourir à des concepts physiques pour décrire cette attente, en l'occurrence bien entendu le concept $\mathrm{d}^{\prime}$ inertie ${ }^{76}$. D'autre part, et en retour, cette attente générale n'est pas spécifiquement psychique et l'on voit que, non seulement par le concept d'inertie mais aussi par le concept d'adaptation, on peut l'étendre au monde organique et physiologique; cela confirme que c'est bien du système corps-esprit-monde que traite Valéry dans ces pages, par opposition aux analyses plus abstraites de l'attention. Enfin, on voit que cette attente générale est tout aussi fondamentale que la fameuse selfvariance dont nous parlions plus haut, et qu'elle est même plus originaire qu'elle s'il est vrai que toute variation, a fortiori la mienne, suppose un fond de permanence pour être saisie comme telle. Nous laisserons de côté les notations dans lesquelles Valéry se demande comment l'on peut instituer volontairement une attente spéciale par distinction d'avec sa forme générale, car elles reviennent à prolonger les analyses relatives à l'attention, comme on le comprend aisément.

En revanche, il nous paraît essentiel de nous arrêter sur le rôle que Valéry reconnaît au souvenir dans la constitution de cette continuité, dans la mesure où ce rôle est structurellement ambigu: dans une proximité thématique parfois surprenante avec certaines analyses génétiques husserliennes ${ }^{77}$, Valéry montre que le souvenir est le lieu où continuité et discontinuité se constituent simultanément, où

${ }^{73} \mathrm{Ibid} .$, respectivement p. 284 et p. 341.

${ }^{74} \mathrm{Ibid.,}$ p. 286. Cf. aussi p. 257, p. 261.

${ }^{75}$ Ibid., p. 262. Cf. aussi p. 343.

${ }^{76}$ Cf. par ex. ibid., p. 326, p. 353.

77 Cf. par ex. E. Husserl, Manuscrits de Bernau sur la conscience du temps (1917-1918), trad. J.-F. Pestureau et A. Mazzù, Grenoble, Jérôme Millon, 2010, p. 278 sq. (Hua XXXIII, p. 361 sq.) ; De la synthèse passive. Logique transcendantale et constitutions originaires, trad. B. Bégout et J. Kessler, Grenoble, Jérôme Millon, 1998, p. 253 sq. (Hua XI, p. 192 sq.), p. 308-315 (Hua XI, p. 266-275), Appendice VIII, p. 350 sq. (Hua XI, p. 365 sq.), Appendice X, p. 368 sq. (Hua XI, p. 384 sq.). 
attente et surprise s'articulent, voire se confondent. D'un côté, en effet, le souvenir est ce qu'on pourrait appeler un agent de la continuité fondamentale : en tant que «sentiment de non-nouveau », il est tout d'abord « un élément du continuum ». " Donc, poursuit Valéry, une adaptation est comparable à la construction immédiate du souvenir ${ }^{78}$. $S^{\prime}$ adapter, $c^{\prime}$ est en quelque sorte se souvenir au présent du présent. C'est la raison pour laquelle Valéry, passant à la réciproque, affirme que « le souvenir assure une continuation - une continuité ${ }^{79}$. D'un autre côté, pourtant, on peut affirmer que «tout souvenir est une surprise élémentaire $\gg^{80}$ au sens où le souvenir est le premier lieu de rupture possible de la continuité de fond, justement parce qu'il s'agit du lieu même où se constitue cette continuité. Sa puissance d'interruption de la continuité est le revers exact de sa puissance de constitution de la continuité, sachant que la mémoire a pour effet notable de produire cumulativement l'oubli du souvenir. C'est bien entendu chez Proust et dans sa mise en scène du souvenir involontaire qu'on peut voir la meilleure illustration de ce que Valéry nomme ici le « souvenir interruptif $»^{81}$.

II. 3. C'est donc le souvenir qui nous permet d'en venir enfin à la surprise, que Valéry nomme aussi la brusquerie et dont nous relèverons ici quatre aspects essentiels.

En premier lieu, il faut revenir sur la puissance de révélation de la surprise relativement au phénomène général de l'attente, «cette prévision continuelle que démontre en la déjouant la surprise ». Les notations convergentes sont nombreuses: "c'est la surprise même qui décèle l'existence de ce qu'elle rompt et met en défaut », si bien qu'on peut ériger en principe que « là où une surprise se produit, il $\mathrm{y}$ avait une attente ${ }^{82}$. Toute attention portée à l'attente est ainsi motivée et rendue possible par sa rupture, mais on aurait tort d'en tirer la conclusion qu'elle serait nécessairement rétrospective. Car je peux aussi observer le phénomène de la surprise en décidant de la provoquer volontairement, et ce non pas pour autrui mais pour moimême ; mais pour que cela arrive et réussisse, il faut introduire une précision que Valéry qualifie de «capitale: on ne peut produire de mouvement brusque volontairement que moyennant une préparation

\footnotetext{
${ }_{78}$ CI, XI, p. 320.

${ }^{79}$ Ibid., p. 327.

${ }^{80}$ Ibid., p. 290, p. 306.

${ }^{81} \mathrm{Ibid} .$, p. 300.

82 Ibid., respectivement p. 352, p. 305 et p. 339.
} 
sensible, de durée finie ${ }^{83}$. Autrement dit, si je veux me surprendre, il faut que je commence par me mettre en situation d'attente. Ce n'est donc pas tant que l'attente précède la surprise qu'elle ne la conditionne, mais le conditionnement est alors réciproque, comme l'atteste ce raisonnement par l'absurde: "Suppose que la surprise n'existe pas. [...] L'attente n'existerait pas. Ce qui est absurde, impossible. Il faut que j'aie de quoi répondre toujours, et que ce toujours soit illusoire, puisse être nargué par le fait $»^{84}$.

En second lieu, si certaines notations valéryennes se plaisent manifestement à souligner les paradoxes temporels créés par l'antériorité réciproque d'une attente tournée vers l'avenir et de sa déception, c'est que la surprise bouleverse en réalité notre expérience "normale » du temps. Rappelons-nous en effet que le temps véritablement significatif n'est pas pour Valéry la succession formelle de nos états mais un effet de notre attention fixant notre variation. Il faut donc s'attendre à ce que la prise en défaut de cette attention spontanée qu'est l'attente produise des effets sur notre temporalité vécue. Et c'est à description de ce bouleversement que s'attachent de nombreuses remarques. Partons du fait que dans la surprise, je fais l'expérience de quelque chose d'inattendu, c'est-à-dire dont les conditions d'apparition dans mon expérience ne pouvaient être anticipées sur la base de mes expériences passées. Valéry décrit cette situation en disant que dans la surprise, j'ai l'effet avant la cause, le tout avant la partie, l'après avant $1^{\prime}$ avant ${ }^{85}$. Cette constatation débouche sur plusieurs descriptions convergentes de la surprise dans ses rapports avec l'attente. Tout d'abord, Valéry affirme que la surprise vient bouleverser la «structure chronologique » du vivant en remettant en question l'enchaînement formel d'un avant et d'un après absolus ${ }^{86}$. C'est pourquoi je suis « saisi » par la surprise, expression sur laquelle Valéry s'arrête et qu'il oppose à la saisie qu'opère de façon symétrique l'attente en tant qu'anticipation : je suis " saisi avant d'avoir saisi » ${ }^{87}$. Donc soit je devance soit je suis devancé ; mais si dans la surprise je suis devancé par l'événement qui m'arrive avant ses conditions, c'est bien en un sens que je suis en retard sur lui. Or à partir de ce concept de retard, toute une série de caractérisations sont développées par Valéry : si je suis en retard c'est que j'ai perdu du temps ; la surprise devient donc l'équivalent du temps perdu et l'attente du temps gagné sur ce qui

\footnotetext{
83 Ibid., p. 359.

${ }^{84} \mathrm{Ibid} .$, p. 316 (et p. 292).

${ }^{85}$ Cf. ibid., respectivement p. 292, p. 312, p. 274.

${ }^{86}$ Cf. ibid., p. 274, p. 314.

87 Ibid., p. 281, p. 342.
} 
arrive. Ou encore, si je suis en retard c'est que j'ai été pris de vitesse ; et si, dans la vie l'esprit comme sur la route, c'est la grande vitesse qui multiplie les occasions d'accident ou d'imprévus, alors l'attente et l'attention pourront être décrites comme des fonctions de ralentissement, grâce auxquelles «tout peut devenir fonctionnel », même si «tout ne peut pas être ralenti, ni donc tout passer de l'accidentel au fonctionnel ${ }^{88}$. La surprise demeure donc inéliminable, au point que la sagesse pratique culmine probablement dans la maxime paradoxale conseillant de s'attendre à être surpris, qu'il s'agisse du monde, des autres ou de la vie elle-même. Quoi qu'il en soit, tous ces éléments descriptifs plus ou moins analogiques sont condensés par Valéry dans un passage important :

Je regarde la surprise comme retard des modifications cachées qui limitent, définissent, localisent, machinent, spécialisent, etc. une perturbation donnée. [...]

C'est un désordre des actes cachés - donc un temps perdu tandis que la préparation correspond à un temps gagné.

C'est donc une attente négative que la surprise qui finit par la perception au lieu de commencer par elle ${ }^{89}$.

Une relation symétrique se construit ainsi entre attente et surprise à partir des effets temporels de la surprise, relation éloquemment confirmée par la notation suivante, qui fournit sur cette base une sorte de définition symétrique : "Ce qui est (déjà) n'est pas (encore) - voici la Surprise. Ce qui n'est pas (encore) est (déjà) - voilà

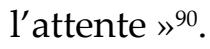

$\mathrm{Qu}$ on puisse ainsi parvenir à une définition générale de la surprise ne doit pourtant pas faire croire qu'il s'agit d'un phénomène uniforme. Au contraire, en troisième lieu, parallèlement à ces essais de définition générique, les analyses valéryennes s'orientent parfois en direction d'une typologie distinguant des formes fondamentales de la surprise. Dans cette perspective, l'aspect quantitatif de la surprise, lié à son degré d'intensité, est jugé insignifiant ; $c^{\prime}$ est un critère qualitatif qui est mis en avant, lié à la question de savoir si la surprise est ou non fonction de l'état particulier dans lequel je me trouve. Il y a donc deux types fondamentaux de surprise, dont la dualité répond à celle de l'attente, soit générale soit spécifique c'est-à-dire déterminée par

\footnotetext{
${ }^{88}$ Ibid., p. 308.

${ }^{89}$ Idem.

${ }^{90}$ Ibid., p. 315.
} 
un certain type d'attention: Valéry parlera de surprise relative pour décrire celle qui est le corollaire de cette concentration exclusive de mon attention sur un objet ou un aspect de l'expérience qui me rend inattentif à ce qui peut dès lors venir me surprendre, et il nommera surprise absolue celle qui est indépendante de l'état particulier de mon attention et qui correspond donc aux expériences plus violentes de ruine des certitudes ou de pertes des repères ${ }^{91}$, bref à ce qu'on appelle le traumatisme, même si ce dernier concept n'apparaît nulle part sous sa plume. Soucieux de rendre compte de la façon la plus précise de cette dualité fondamentale, Valéry convoque parfois d'autres oppositions conceptuelles pour la décrire, comme celle du potentiel et de l'actuel : en effet, le traumatisme de la surprise absolue correspond à l'expérience où «ce que je croyais impossible se produit $»^{92}$, tandis que la surprise relative vient du fait qu'une possibilité que j'avais temporairement exclue se rappelle à mon attention. Si donc la surprise concerne dans tous les cas la modalité du possible, ce n'est pas toujours selon la même signification. Alors que la surprise relative rappelle à la conscience l'actualité de quelque chose qui avait été écarté comme "simple» possible, dans le cas de la surprise absolue, "c'est le possible qui est ébranlé ${ }^{93}$ comme tel. En d'autres termes, il est impossible de s'attendre à un traumatisme, et c'est pourquoi Valéry peut écrire que cette forme de surprise correspond au manque d'un certain potentiel tandis que l'autre, relative, correspond au manque $\mathrm{d}^{\prime}$ un certain actuel ${ }^{94}$. Enfin, dans la mesure où la différence entre ces deux types fondamentaux de la surprise renvoie à l'opposition entre une surprise indépendante de tout état particulier dans lequel je me trouverais et une surprise consécutive à l'un de ces états, il arrive à Valéry d'user de sa distinction fondamentale entre le formel et le significatif pour les caractériser ${ }^{95}$. Ainsi la surprise absolue est-elle surprise formelle, pouvant avoir lieu « à tout instant quelconque $»^{96}$ sans qu'aucun instant soit plus signifiant qu'un autre relativement à la possibilité de cette surprise et sans que l'idée d'une préparation ou $\mathrm{d}^{\prime}$ une anticipation ait le moindre sens ; la surprise relative est quant à elle significative, puisque sa possible irruption est une variable (je peux $\mathrm{m}^{\prime} \mathrm{y}$ préparer ou non) induite par un certain état de mon esprit

\footnotetext{
${ }^{91}$ Ibid., p. 357-358.

92 Ibid., p. 357.

${ }^{93} \mathrm{Ibid} .$, p. 354.

${ }_{94}$ Cf. ibid., p. 315.

${ }^{95}$ Cf. ibid., p. 347.

${ }_{96}$ Ibid., p. 354 (cf. aussi p. 357).
} 
(qu'il soit actif comme la concentration attentive, ou passif comme le sommeil).

Mais un dernier aspect de l'analyse valéryenne de la surprise reste à évoquer, qui nous reconduira, en guise de conclusion, à la signification de l'entreprise même des Cahiers. En effet, Valéry est attentif au fait que si la surprise prend l'attente en défaut et la révèle par la même comme sa condition, elle la provoque aussi en guise de réaction sous la forme d'un (r)éveil de l'attention. C'est ce qu'on peut apercevoir à partir de la description de la surprise comme choc, dû à « l'arrivée $\mathrm{d}^{\prime}$ un événement non compris dans le champ de substitution $\gg^{97}$, c'està-dire qui déjoue une certaine fixation, une certaine coordination (au sens des axes de coordonnées) du champ de variabilité qu'une attention ou une attente a préalablement instituée. Or ce choc a pour effet immédiat un regain d'attention que Valéry pointe à travers des expressions comme «je deviens tout oreilles; tout yeux $»^{98}$. Mais derrière ce « je deviens » il y a tout un processus que Valéry nomme $l^{\prime}$ " oscillation » consécutive au choc et dont il propose la description suivante :

Quand ce qui survient ne peut succéder à ce qui était, quand il n'est pas compris dans le domaine du système sensible réciproque de ce qui était, il y a oscillation. Au moment même, il y a sensation brute sans figure, puis retour par phénomènes alternants ${ }^{99}$.

En quoi consiste ce retour? Essentiellement en une stratégie mentale de réinscription de ce qui a interrompu la continuité dans la continuité elle-même : il s'agit d'accommoder après l'éblouissement, de comprendre en com-prenant, notamment par le moyen de la répétition dont l'effet consiste à neutraliser la nouveauté de la surprise (répéter un mot surprenant, un vers qu'on apprend, revivre une situation ou un événement). Comme l'écrit Valéry dans l'une des seules descriptions précises qu'il en donne:

Si le choc n'a pas été trop intense, on voit se faire alors un travail qui a pour... but de passer du point antérieur au présent, sans surprise, c'est-à-dire en s'attendant cette fois, à l'événement surprenant, et en le ravivant à froid, avec une réponse prête ${ }^{100}$.

\footnotetext{
${ }^{97}$ Ibid., p. 283.

${ }_{98}$ Ibid., p. 311.

${ }_{99} \mathrm{Ibid.,}$ p. 306. Cf. également VIII, p. 160 ; XI, p. 289, p. 347, p. 353.

100 Ibid., p. 361.
} 
Ainsi, dans la mesure où ce retour en arrière revient à mettre en place après-coup la structure d'attente qui a fait défaut ou a été prise en défaut, il s'agit au fond d'une stratégie de récupération de l'événement surprenant dont le premier effet est bien entendu de le faire disparaître comme tel. Ainsi, même si l'attente est bien en un sens un effet de la surprise, cette dernière est en définitive un événement de l'attente, qui se restaure et se réinstaure moyennant la surprise. Mais dans la mesure où cette stratégie de récupération permet à l'esprit de s'approprier après-coup ce qui l'a surpris, elle est simultanément pour lui une (re)prise de possession de soi, une restauration de soi, raison pour laquelle Valéry note que « la surprise me transforme en moi-même »101.

Ces analyses invitent donc, dans leur ensemble, à ne pas réduire abstraitement l'expérience de la surprise à son premier moment, celui du choc, de la stupeur et de la dépossession ; envisagée concrètement, dans toutes ses dimensions, elle est tout autant et à la fois occasion répétée de réappropriation de soi et $\mathrm{du}$ monde. C'est pourquoi il faudrait peut-être parler en définitive de la surprise comme d'une ponctuation dans la vie de l'esprit, qui la rythme et la scande plutôt qu'elle ne la fige, qui en prolonge et en entretient le dynamisme plutôt qu'elle ne l'interrompt.

D'un autre côté, si le moi lui-même est en jeu dans toute expérience de surprise, se constituant pour soi et se réinstituant à l'occasion de chacune d'entre elles, alors les expériences dans lesquelles il se surprend lui-même prennent inévitablement un relief tout particulier; non pas tant, à vrai dire, celles dans lesquelles il est surpris par soi $^{102}$ que celles dans lesquelles il est surpris de soi. De fait, nombreuses sont les notations des Cahiers qui autorisent à voir dans ce type particulier d'auto-surprise le véritable thaumazein valéryen: l'étonnement du moi devant lui-même, devant son ipséité et devant son inscription mondaine - l'étonnement d'être soi dans le monde ${ }^{103}$. Et c'est peut-être parce que cet étonnement réflexif est fondamentalement double (il est à la fois étonnement d'être un moi - ou le moi - et étonnement d'être ce moi) que Valéry est tenté d'y voir la surprise par excellence : «Apparaître soi-même, c'est la surprise »104. Mais si c'est bien le cas, c'est probablement parce qu'une expérience de ce

\footnotetext{
101 Ibid., p. 354.

102 Cf. ibid., p. 318, p. 351.

${ }^{103}$ Cf. C, XII, p. 190 : «Quel étonnement muet que tout soit et que moi je sois ».

${ }^{104}$ CI, XI, p. 310 ; cf. aussi p. 356.
} 
type est à l'origine de l'entreprise entière des Cahiers. En effet, s'il est bien connu que cette dernière procède d'un «coup d'État» intellectuel ${ }^{105}$ lui-même consécutif à la crise de la « nuit de Gênes » d'octobre $1892^{106}$, il ne nous paraît pas déraisonnable de considérer la rédaction des Cahiers comme l'immense oscillation consécutive à un choc initial (dont la nuit de Gênes n'est peut-être elle-même qu'une conséquence, un contrecoup ou une réplique) qui ne fut autre pour Valéry que la surprise devant soi, la surprise d'être soi, celui qu'on est, avec ses défauts et ses limites. Prendre possession de soi, s'approprier soi-même «tel quel» par un exercice répété de l'attention qui réduise cette étrangeté ou étrangèreté initiale dans l'acte même par lequel il découvre et modélise les lois du fonctionnement de l'esprit ${ }^{107}$, c'est peut-être la folle ambition des Cahiers.

${ }^{105}$ C, VIII, p. 762 (repris in Cahiers, II, p. 460).

${ }^{106}$ Sur cet épisode fondateur et son caractère plus ou moins légendaire, cf. M. Jarrety, Paul Valéry, op. cit., p. 112-120.

${ }_{107}$ Cf. C, XII, p. 392 : «Un jour que je n'en pouvais plus, j'ai pris la décision de m'accepter tel $q u e l$. J'avais 20 ans. J'ai résolu et tenu de mesurer mes pouvoirs dans le silence et de me borner à cet exercice secret...» (repris in Cahiers, I, p. 111). Sur le thème, décisif et omniprésent, de l'étrangeté à soi, cf. par ex. C, XXVIII, p. 125 (repris in Cahiers, I, p. 224). 